\title{
Targeted Programming of the Lymph Node Environment Causes Evolution of Local and Systemic Immunity
}

\author{
James I. Andorko, ${ }^{1}$ Joshua M. Gammon, ${ }^{1}$ Lisa H. Tostanoski, ${ }^{1}$ Qin Zeng, ${ }^{1}$ \\ and Christopher M. Jewell ${ }^{1,2,3}$ \\ ${ }^{1}$ Fischell Department of Bioengineering, University of Maryland, 8228 Paint Branch Drive, College Park, MD 20742, USA; \\ ${ }^{2}$ Department of Microbiology and Immunology, University of Maryland School of Medicine, 685 West Baltimore Street, \\ Baltimore, MD 21201, USA; and ${ }^{3}$ Marlene and Stewart Greenebaum Cancer Center, 22 S. Greene St., Baltimore, MD 21201, \\ USA
}

(Received 18 February 2016; accepted 16 June 2016; published online 27 June 2016)

Associate Editor Michael R. King oversaw the review of this article.

\begin{abstract}
Biomaterial vaccines offer cargo protection, targeting, and co-delivery of signals to immune organs such as lymph nodes (LNs), tissues that coordinate adaptive immunity. Understanding how individual vaccine components impact immune response has been difficult owing to the systemic nature of delivery. Direct intra-lymph node (i.LN.) injection offers a unique opportunity to dissect how the doses, kinetics, and combinations of signals reaching LNs influence the LN environment. Here, i.LN. injection was used as a tool to study the local and systemic responses to vaccines comprised of soluble antigen and degradable polymer particles encapsulating toll-like receptor agonists as adjuvants. Microparticle vaccines increased antigen presenting cells and
\end{abstract}

Address correspondence to Christopher M. Jewell, Fischell Department of Bioengineering, University of Maryland, 8228 Paint Branch Drive, College Park, MD 20742, USA. Electronic mail: cmjewell@umd.edu

James I. Andorko and Joshua M. Gammon have contributed equally.

Christopher M. Jewell is an Assistant Professor in the Fischell Department of Bioengineering at the University of Maryland and a Damon Runyon-Rachleff Innovator. Dr. Jewell completed his Ph.D. in Chemical Engineering with Professor David Lynn in 2008 at the University of Wisconsin. He then joined the Boston Consulting Group, working in R\&D strategy for global pharmaceutical and biotechnology clients. Dr. Jewell began his postdoctoral training in 2009 as a Ragon Fellow in Professor Darrell Irvine's Lab at MIT, and held a concurrent appointment in the Division of Vaccine Research at Harvard. Chris launched his lab in 2012 to investigate the interactions between synthetic materials and lymph nodes, and to harness these interactions for therapeutic vaccination. Dr. Jewell has authored 41 papers and patents, including reports in ACS Nano, $P N A S$, and Nature. His efforts have been recognized by numerous honors, including the NSF CAREER Award, Young Investigator Awards from the Alliance for Cancer Gene Therapy and the Melanoma Research Alliance, and the University's Research Scholar Award. In 2012 Chris appeared in USA Today as a "New Face of Engineering", and was recently named the state of Maryland's Outstanding Young Engineer, the state's highest professional honor awarded to an engineer under 36.

This article is part of the 2016 Young Innovators Issue. lymphocytes in LNs, enhancing activation of these cells. Enumeration of antigen-specific $\mathrm{CD}^{+} \mathrm{T}$ cells in blood revealed expansion over 7 days, followed by a contraction period over 1 month as memory developed. Extending this strategy to conserved mouse and human tumor antigens resulted in tumor antigen-specific primary and recall responses by $\mathrm{CD}^{+} \mathrm{T}$ cells. During challenge with an aggressive metastatic melanoma model, i.LN. delivery of depots slowed tumor growth more than a potent human vaccine adjuvant, demonstrating local treatment of a target immunological site can promote responses that are potent, systemic, and antigen-specific.

Keywords-Lymph node, Vaccine, Adjuvant, Microparticle and nanoparticle, Cancer, Immunotherapy.

\section{INTRODUCTION}

Historically vaccine design has focused on generating potent, specific immune responses. However, equally important for vaccines aimed at persistent and

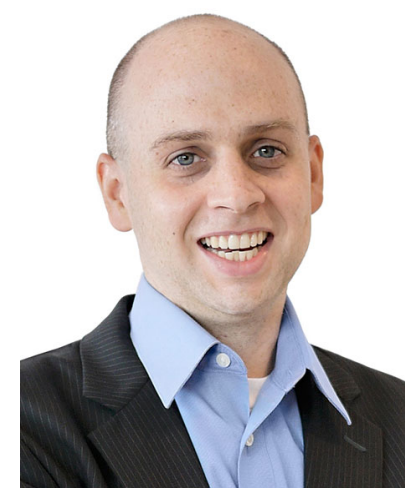


emerging diseases, is the need to better control the nature of the immune responses that are generated. For example, in the context of cancer vaccination, tumor-specific $\mathrm{CD} 8^{+} \mathrm{T}$ cells that exhibit memory-like characteristics and proliferate at very high rates might help overcome the immunosuppressive tumor microenvironment. ${ }^{10,33}$ Even vaccines aimed at well controlled pathogens - such as flu - could benefit from formulations that offer better immunomodulatory capabilities, in this example, by conferring increased production of mucosal antibodies. ${ }^{7}$ Another area of intense research along these lines is in the exploitation of new adjuvants - such as toll like receptor agonists (TLRas) that stimulate pathogen-detecting inflammatory pathways. These molecules can be delivered alone, or in combination to create polarizing or synergistic effects. ${ }^{6,26,50,51,55}$ Better understanding of the effects of specific vaccine components, adjuvants, and carriers, along with knowledge of how these agents work together, would help support the design of more effective vaccines.

Lymph nodes (LNs) are tissues that initiate, maintain, and regulate adaptive immune response, and are thus critical targets for vaccines and immunotherapies. At these sites, antigen presenting cells (APCs) display antigens to $\mathrm{T}$ and $\mathrm{B}$ cells with the same specificity to mount antigen-specific effector function. ${ }^{14}$ Thus the local signals integrated in LNs help define the specificity, magnitude, and nature of the resulting systemic responses. A key hurdle facing new vaccines and immunotherapies is efficiently targeting these sites. ${ }^{30}$ For example, to effectively prime lymphocytes against a specific antigen, both the antigen and an adjuvant or other stimulatory immune signal need to be localized to the same tissue, while the combinations and relative concentrations of vaccine components dramatically impact the characteristics of this response. Unsurprisingly, significant interested has developed in strategies that allow more efficient delivery to LNs and more precise control over the local environment in these tissues.

To address the challenges above, many reports in the past several decades have investigated biomaterial carriers (e.g., polymer particles, ${ }^{25,49}$ liposomes $15,21,32,48$ ) that encapsulate or adsorb combinations of antigens and adjuvants. ${ }^{2}$ The tunable sizes, particulate nature, and ability to co-deliver cargos make these vehicles attractive as vaccine formulations that can be injected and drain to LNs or can be carried there by APCs. ${ }^{18}$ Particle size plays a major role in the efficiency and route by which these vaccines reach $\mathrm{LNs},{ }^{42}$ an area that has been heavily investigated..$^{2,18}$ While many exciting approaches have been reported, even those that generate robust immune responses are limited in the control they provide over the routes or doses by which particles reach LNs after injection. Instead, vaccines generally rely on passive draining through lymphatic vessels, uptake by APCs and subsequent trafficking to LNs, or more recently, active targeting using receptor/ligand interactions. ${ }^{2,18}$ Thus, a relatively small faction of the total injected dose actually reaches LNs, ${ }^{19,42}$ increasing the required dose in some cases, or preventing efficacious response in others. These effects are also important since some vaccine or immunotherapy components have toxic or inflammatory effects that limit the dose or frequency of administration.

A consideration specific to biomaterial carriers is the growing list of polymers, such as poly(lactic-coglycolic acid) (PLGA), polystyrene, and others, ${ }^{2,3,37,47}$ that exhibit intrinsic inflammatory effects even in the absence of other immune signals. ${ }^{2}$ PLGA, for example, is used in countless vaccine and immunotherapy studies, but can activate the inflammasome and increases stimulatory response to TLRas. ${ }^{47}$ While these are characteristics that can be harnessed, they can also complicate vaccine research because of the increased complexity resulting from "carrier-effects" that alters how the immune system responds to antigens or other vaccine components. A better understanding of how immune signals - and their biomaterial carriers - interact with the local LN microenvironment, and how these interactions direct systemic immunity would help improve vaccine performance, while also contributing to more rational vaccine design strategies.

We recently developed a strategy to deposit biomaterial vaccine depots directly in LNs of mice using intra-lymph node (i.LN.) injection. ${ }^{3,4,22}$ This platform allows direct control over delivery of vaccine components to LNs, and sustained release of encapsulated cargo within these tissues. In our previous work, we discovered i.LN. delivery of microparticles (MPs) encapsulating adjuvant generate more potent responses than nanoparticles or soluble adjuvant because these large particles are better retained in LNs. ${ }^{22}$ Therefore, we sought to use i.LN. injection of adjuvant-loaded MPs as a tool to study the evolution of these local and systemic responses over time in mice. We demonstrate that $i . L N$. deposition of vaccine depots consisting of PLGA MPs loaded with a TLR3a and suspended in soluble ovalbumin (OVA) antigen increases the number of APCs and lymphocytes in LNs over the course of 7 days. Treatment does not alter the relative composition of these compartments, but does increase the activation of resident APCs. A single treatment with these vaccine depots expands antigenspecific $\mathrm{CD}^{+} \mathrm{T}$ cells locally in treated LNs and systemically in peripheral blood, evolving from a potent effector response at day 7 to a memory response by day 28. We also show this approach is generalizable: i.LN. 
injection of vaccine depots loaded with either PolyIC or $\mathrm{CpG}$ - potent TLRas being explored in human trials - and then mixed with conserved human melanoma antigens potently expand tumor-specific $\mathrm{CD} 8^{+} \mathrm{T}$ cells. These effects correlate with slowed tumor progression during an aggressive challenge with metastatic melanoma. Together this work demonstrates that local programming of distinct LNs with adjuvant depots can be used to drive local alterations that promote immunity that is systemic and antigen-specific.

\section{MATERIALS AND METHODS}

\section{Particle Synthesis}

Degradable MPs were synthesized via a doubleemulsion, solvent evaporation technique. ${ }^{4,22}$ For lipid stabilized particles, 1,2-dioleoyl-sn-glycero-3-phosphocholine, 1,2-distearoyl-sn-glycero-3-phosphoethanolamine- $N$-[amino(polyethylene glycol)-2000], and 1,2-dioleoyl-3-trimethylammoniumpropane (Avanti Polar Lipids) were prepared at a 60:20:20 mol ratio and dried under nitrogen. $80 \mathrm{mg}$ of PLGA (Sigma) was dissolved with the $5.15 \mu \mathrm{mol}$ of lipids in $5 \mathrm{~mL}$ of dichloromethane. An inner aqueous phase containing $500 \mu \mathrm{L}$ of water or $5 \mathrm{mg}$ of polyinosinic-polycytidylic acid (PolyIC) (Invivogen) in $500 \mu \mathrm{L}$ of water was added to this organic phase containing polymer and lipid and sonicated for $30 \mathrm{~s}$ at $12 \mathrm{~W}$ to form the first emulsion. This emulsion was then added to $40 \mathrm{~mL}$ of water, homogenized for $3 \mathrm{~min}$ at $16,000 \mathrm{rpm}$, and then allowed to evaporate overnight while stirring to remove any excess organic solvent. Particles stabilized with poly(vinyl alcohol) (PVA, Sigma) were formed as above by removing lipids and replacing the second water phase with a $2 \% \mathrm{w} / \mathrm{v}$ solution of PVA. For particles containing $\mathrm{CpG}$ (sequence: $5^{\prime} \mathrm{T}$-C-C-A-TG-A-C-G-T-T-C-C-T-G-A-C-G-T-T 3', IDT), $3 \mathrm{mg}$ of $\mathrm{CpG}$ in $500 \mu \mathrm{L}$ of water was used for the first aqueous phase. After overnight stirring, all particle formulations were passed through a $40 \mu \mathrm{m}$ cell strainer to remove any large aggregates and collected via centrifugation $\left(5000 \times g, 5 \mathrm{~min}, 4^{\circ} \mathrm{C}\right)$. Supernatants were removed and particles were washed three times with $1 \mathrm{~mL}$ of water then suspended in water or PBS for animal studies, or lyophilized and stored at $4{ }^{\circ} \mathrm{C}$ prior to use. For preparation of fluorescently-labeled particles, $5 \mu \mathrm{L}$ of DiI (Invitrogen) was added to the organic phase.

\section{Particle Characterization}

Particle diameter was determined using an LA-950 laser diffraction analyzer (Horiba). Zeta potential was measured using a Malvern Zetasizer Nano ZS90. PolyIC and $\mathrm{CpG}$ loading levels were determined via
$\mathrm{UV} /$ Vis spectrophotometry after hydrolyzing a known mass of lyophilized particles overnight in $0.2 \mathrm{M}$ $\mathrm{NaOH}$. Absorbance values were compared to standard curves of known concentrations of PolyIC or $\mathrm{CpG}$ to determine a mass of cargo per mass of polymer.

\section{i.LN. Injection}

For each animal study, a small region of fur was removed from the lateral hind quarter of 4-6 week old C57BL6 mice (The Jackson Laboratory) by shaving the area and applying a mild depilatory. Tracer dye (Evans Blue) was then injected subcutaneously (s.c.) on each side of the tail base as previously reported. ${ }^{3,4,22}$ After allowing $16 \mathrm{~h}$ for the tracer dye to drain to the inguinal LNs for visualization, a $31 \mathrm{G}$ insulin needle was used to inject $10 \mu \mathrm{L}$ containing the indicated treatment into each inguinal LN. For visualization of particles in LNs, $1 \mathrm{mg}$ of DiI labeled MPs were injected. For model antigen studies, vaccinations consisted of $1 \mathrm{mg}$ of particles encapsulating $\sim 8.5 \mu \mathrm{g}$ PolyIC $/ \mathrm{mg}$ MPs suspended in PBS with $25 \mu \mathrm{g}$ soluble ovalbumin (OVA, Worthington) ('PolyIC MP/OVA'), an injection of $1 \mathrm{mg}$ of PLGA MPs with no cargo ('Empty'), or an injection of buffer alone ('sham'), as indicated. In experiments comparing PolyIC and $\mathrm{CpG}$ depots, equivalent doses of adjuvant encapsulated in MPs were administered i.LN, after being suspended in PBS with $25 \mu \mathrm{g}$ of soluble OVA or soluble Trp2 (SVYDFFVWL, Genscript) antigens. After priming, mice were boosted with soluble vaccine treatments s.c. at each side of tail base at day 21 , with each injection consisting of $25 \mu \mathrm{g}$ antigen and $25 \mu$ g adjuvant. For studies comparing melanoma antigens (Trp2, hgp100), treatments included $1 \mathrm{mg}$ of particles containing $\sim 3.5 \mu \mathrm{g} \mathrm{CpG} / \mathrm{mg}$ MPs suspended in PBS with $25 \mu \mathrm{g}$ of soluble Trp2 ('CpG MP/Trp2') or soluble hgp100 (KVPRNQDWL, Genscript; 'CpG $\mathrm{MP} / \mathrm{hgp} 100^{\prime}$ ) antigens, or strong pre-clinical vaccine consisting of $50 \mu \mathrm{g}$ of $\mathrm{CpG}$ and $50 \mu \mathrm{g}$ peptide formulated with montanide ISA 51 (Seppic; 'Montanide/ $\mathrm{CpG} / \operatorname{Trp2}$ ' or 'Montanide/CpG/hgp100'). After vaccinating $i . L N$. at day 0 , subsequent boosts for MP groups were given at days 15 and 36 post prime and were identical to the prime but administered s.c. at the tail base. For the montanide groups, all injections were s.c., but the second boost consisted of soluble Trp2 or soluble hgp100 mixed with CpG (see caption). All animal studies were approved by the University of Maryland IACUC and conducted in accordance with local, state, and federal guidelines.

\section{Tissue Collection, Processing, and Flow Cytometry}

At the indicated times after treatment, LNs were collected from mice, placed in PBS, and processed into 
single cell suspensions by mechanical dissociation through a $40 \mu \mathrm{m}$ strainer. Cells were split into three portions. One portion of cells was centrifuged $(800 \times g$, 5 min, $\left.4{ }^{\circ} \mathrm{C}\right)$ and suspended in FACS buffer $(1 \times$ PBS with $1 \% \mathrm{w} / \mathrm{v}$ bovine serum albumin, Sigma) containing $1 \%$ DAPI (Invitrogen) and Liquid Counting Beads (BD) to quantify cell viability and enumerate total cell numbers using a FACS Canto II (BD), respectively. The other two portions of cells were washed once with $1 \mathrm{~mL}$ of FACS buffer then blocked with Fc Block (anti$\mathrm{CD} 16 / \mathrm{CD} 32, \mathrm{BD})$ for $10 \mathrm{~min}$ at room temperature to inhibit any non-specific binding. After blocking, one portion of cells was stained for innate cell type and activation with indicated antibodies against cell surface markers including CD11c, F4/80, CD40, CD80, CD86, and I-A/I-E (mouse MHCII). Cells were then washed twice, suspended in FACS buffer, and quantified via flow cytometry. The final portion of cells was stained for lymphocyte populations and antigen-specific tetramer levels. First, $25 \mu \mathrm{L}$ of anti-SIINFEKL tetramer was added and incubated for $30 \mathrm{~min}$ at room temperature. Then, $25 \mu \mathrm{L}$ of antibodies against surface markers including B220, CD3, CD4, and CD8 were added and incubated for $20 \mathrm{~min}$ at room temperature. Cells were then washed and evaluated, as above. The frequency of each cell population (percent of parent population) and number of counted cells per identical acquisition volume $(80 \mu \mathrm{L})$ was evaluated. The B220 antibody was purchased from eBiosciences and all other antibodies were purchased from BD.

\section{MHC Tetramer Staining of Peripheral Blood}

Every 7 days, $100 \mu \mathrm{L}$ of blood was collected from mice treated as above via submandibular bleeding. Red blood cells were removed by adding $1 \mathrm{~mL}$ of ACK lysis buffer to the blood, incubating for $3 \mathrm{~min}$, collecting cells via centrifugation $\left(800 \times g, 5 \mathrm{~min}, 4{ }^{\circ} \mathrm{C}\right)$, and repeating with $1 \mathrm{~mL}$ of fresh ACK lysis buffer. After the second round of ACK lysis buffer, cells were suspended in FACS buffer, blocked with Fc Block, and stained with a tetramer specific for either SIINFEKL (CD8-epitope of OVA), Trp2, or hgp100 for $30 \mathrm{~min}$ at room temperature. All tetramers were purchased from MBL International. Following incubation, cells were stained against surface markers CD3, CD8, CD44, and CD62L for $20 \mathrm{~min}$ at room temperature. After washing twice with FACS buffer, cells were suspended in FACS buffer containing DAPI and the percentage of antigen-specific cytotoxic $\mathrm{T}$ cells $\left(\mathrm{DAPI}{ }^{-}, \mathrm{CD} 8^{+}\right.$, tetramer $^{+}$) was quantified via flow cytometry. To determine generation of central memory $\mathrm{T}$ cell phenotypes, tetramer ${ }^{+} \mathrm{CD}^{+}$cells were gated for $\mathrm{CD} 44^{\text {high }} /$ CD62 $\mathrm{L}^{\text {high }}$ populations and compared to the percentage of effector memory $\mathrm{T}$ cells $\left(\mathrm{CD} 44^{\text {high }} / \mathrm{CD} 62 \mathrm{~L}^{\text {low }}\right)$.

\section{Tumor Challenge Studies}

In some studies, after treating mice with the indicated vaccines, mice were administered 300,000 B16-F10 cells (ATCC) in $100 \mu \mathrm{L}$ of $1 \times$ PBS s.c. at the hind flank. Each day following inoculation, body weight was monitored and tumor burden was calculated as a product of two orthogonal diameters. Mice were euthanized according to IACUC-approved humane endpoints when the aggregate tumor burden reached $150 \mathrm{~mm}^{2}$.

\section{Immunohistochemical Analysis}

At indicated time points, inguinal LNs were removed and frozen in OCT compound (Tissue-Tek). Using a Microm HM 550 cryostat (Thermo Fisher Scientific Inc.), $6 \mu \mathrm{m}$ sections of LNs were cut, transferred to slides, and allowed to dry overnight. LN tissue was then fixed for $5 \mathrm{~min}$ in ice-cold acetone then washed in $1 \times$ PBS. Samples were then blocked for non-specific binding of secondary antibody using 5\% goat and 5\% donkey serum in $1 \times$ PBS for $30 \mathrm{~min}$. After washing in PBS, tissues were stained for cell surface markers including B220 (eBioscience), CD3 (Abcam), and CD11c (BD) for $1 \mathrm{~h}$ at room temperature. After washing twice with PBS, fluorescent secondary antibodies (Jackson Immunoresearch) were added for $45 \mathrm{~min}$ then washed three more times. After staining, sections were fixed with $4 \%$ paraformaldehyde, washed with PBS, quenched with $1 \%$ glycerol in PBS, and washed again before mounting in Prolong Diamond Antifade Mountant (LifeSciences) and imaging using an Olympus IX83 fluorescent microscope. Processing of images was conducted versus an antibody iso-type control and levels were adjusted equally for all similar channels.

\section{Statistical Analysis}

Student's $t$ tests were used in comparison of two groups. One-way ANOVA with a Tukey post-test was used to compare three or more groups, or two-way ANOVA for comparisons over time. In all cases, analyses were carried out with Graphpad Prism (version 6.02). Error bars in all panels represent the mean \pm SEM and $p$ values $\leq 0.05$ were considered significant. Levels of significance were defined as ${ }^{*} p<0.05 ; * * p<0.01 ;{ }^{* * *} p<0.001 ; * * * * p<0.0001$.

\section{RESULTS}

\section{PLGA MPs are Dispersed in LNs Following i.LN. Injection}

PLGA MPs were synthesized via a double-emulsion/solvent evaporation technique allowing for the 
inclusion of negatively charged nucleic acid TLRa adjuvants PolyIC or CpG with loading levels of $8.5 \mu \mathrm{g} /$ mg MP or $3.5 \mu \mathrm{g} / \mathrm{mg}$ MP, respectively (Table 1 ). Addition of PolyIC led to an increase in particle diameter from 2.2 to $4.3 \mu \mathrm{m}$ and a shift in zeta potential from $24.9 \mathrm{mV}$ to $-23.7 \mathrm{mV}$; replacement of PolyIC with $\mathrm{CpG}$ led to similar shifts (Table 1). To first confirm retention of injected MPs into LNs, we injected DiI-labeled MPs into inguinal LNs of mice using the approach we previously described (Fig. 1a). 3,4,22 28 days after injection, LNs were removed and then stained for B cell (Fig. 1b, cyan) and $\mathrm{T}$ cell zones (Fig. 1b, white). Fluorescent microscopy confirmed retention of MPs in the LNs at this time point (Fig. 1b, green).

TABLE 1. Characteristics of adjuvant loaded PLGA-MPs used in $i . L N$. injection studies.

\begin{tabular}{lccr}
\hline & Diameter $(\mu \mathrm{m})$ & $\begin{array}{c}\text { Loading } \\
(\mu \mathrm{g} \text { cargo/mg MP })\end{array}$ & $\begin{array}{c}\text { Zeta Potential } \\
(\mathrm{mV})\end{array}$ \\
\hline Empty & $2.19 \pm 0.14$ & $\mathrm{n} / \mathrm{a}$ & $24.93 \pm 0.91$ \\
PolylC & $4.26 \pm 0.09$ & $8.53 \pm 0.46$ & $-23.70 \pm 0.71$ \\
CpG & $4.02 \pm 0.14$ & $3.45 \pm 0.37$ & $-23.23 \pm 2.54$ \\
\hline
\end{tabular}

(a)

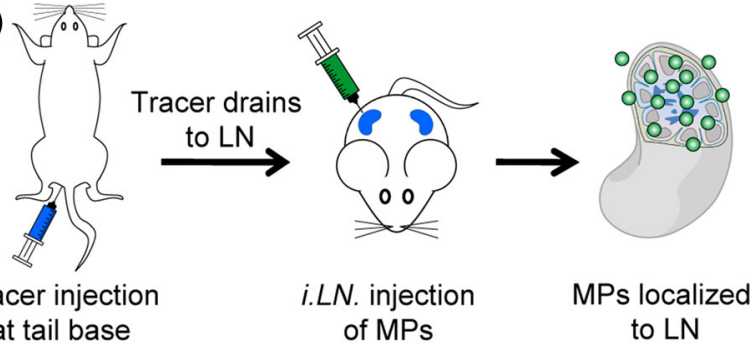

(b)

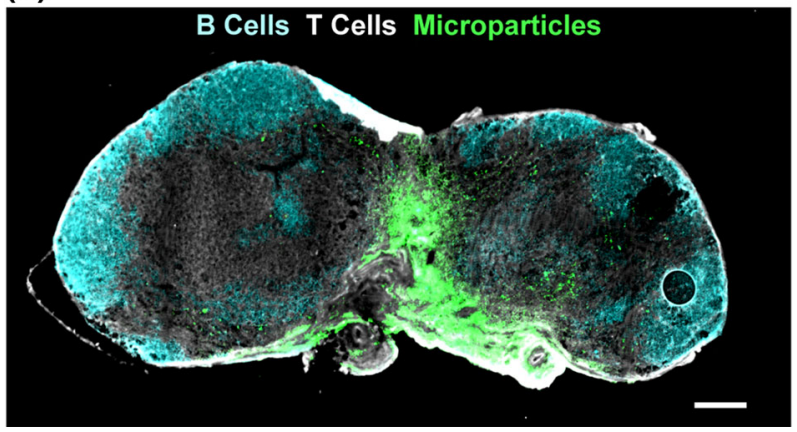

FIGURE 1. Vaccine depots can be locally deposited in LNs via i.LN. injection. (a) Schematic depicting i.LN. injection of vaccine depots. A tracer dye is injected s.c. at the tail base, which then drains to the inguinal LNs allowing visualization of the LN through the skin. Vaccine depots can then be injected into the LN; (b) Histological section of LN 28 days after i.LN. injection of fluorescent depots. B cells $\left(\mathrm{B220}^{+}\right.$, cyan), $\mathrm{T}$ cells (CD3 $^{+}$, white), PLGA MPs (Dil, green). Scale bar $=200 \mu \mathrm{m}$.

\section{i.LN. Injection of PolyIC MP/OVA Increases the Number of APCs and Lymphocytes in LNS}

After confirming MPs are retained in LNs of mice over 4 weeks, we used i.LN. injection to administer a vaccine of PolyIC MPs mixed with soluble OVA (PolyIC MP/OVA), or to administer a buffer injection (sham). Cell viability and the frequency and number of DCs, macrophages, $\mathrm{T}$ cells, and $\mathrm{B}$ cells in the treated nodes were then monitored over 1 week using identically-treated sets of groups. Following treatment, PolyIC MP/OVA, while slightly diminishing initial cell viability relative to sham, did not impact viability after 1 week (Fig. 2a). Particles did cause an increase in the overall number of cells (Fig. 2b), as well as the volume
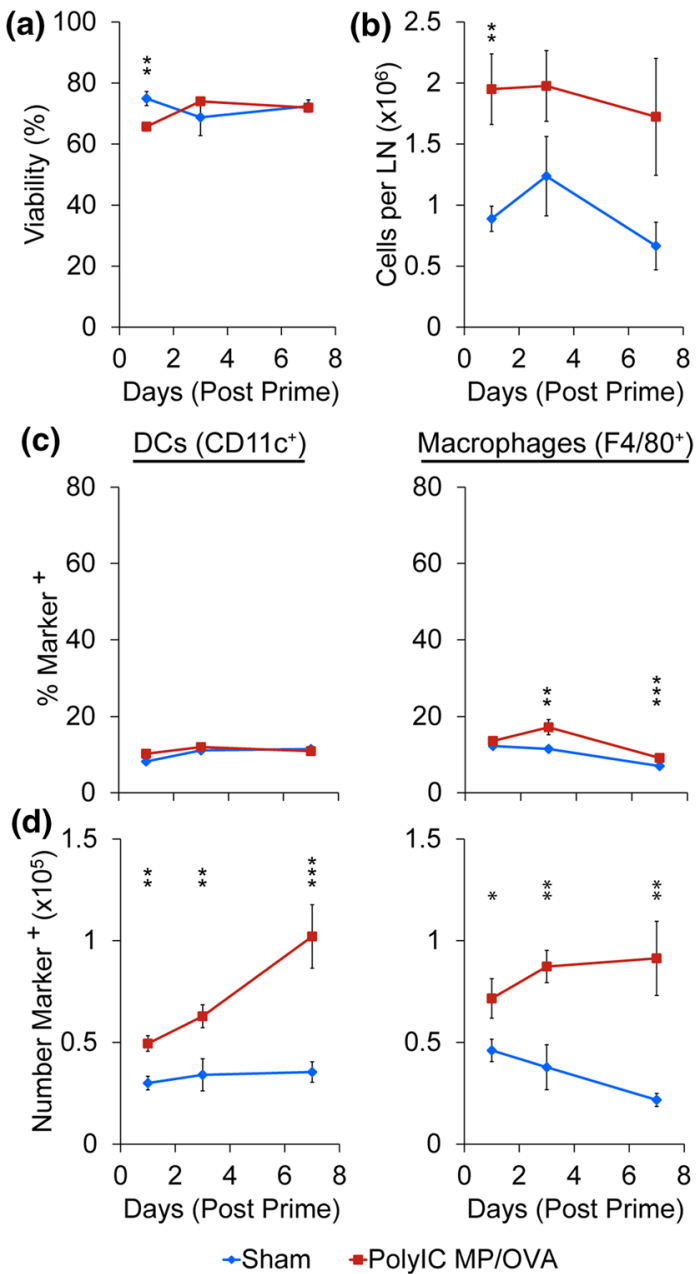

FIGURE 2. i.LN. injection of PolyIC MP/OVA depots increases innate cell numbers in the LNs without affecting cell viability. (a) Viability and (b) total number of LN cells after $i . L N$. injection of PolyIC MP/OVA depots or a sham injection of PBS at days 1, 3, and 7. (c) Percentage of total LN cells which are DCs $\left(\mathrm{CD} 11 \mathrm{c}^{+}\right)$and macrophages $\left(\mathrm{F} 4 / 80^{+}\right)$and (d) number of DCs and macrophages in LNs counted in an identical acquisition volume $(80 \mu \mathrm{L}) . n=9-10 \mathrm{LNs}$ per group with bars depicting mean \pm SEM. $\left({ }^{\star} p<0.05 ;{ }^{* *} p<0.01 ;{ }^{* \star} p<0.001\right)$. 

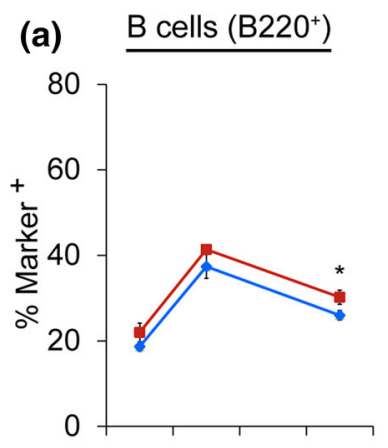

(b)

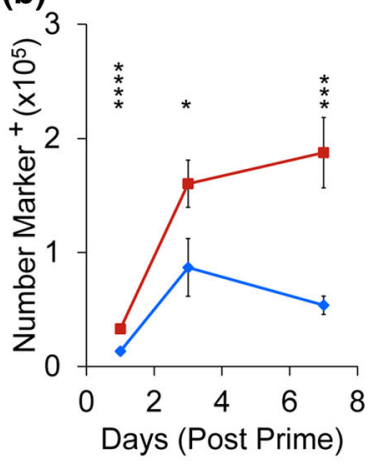

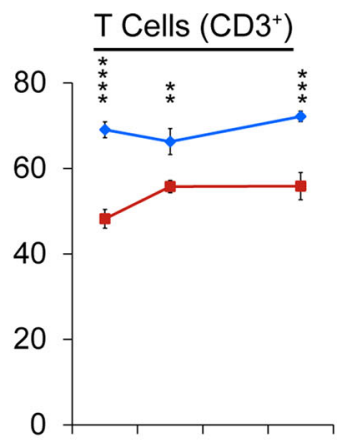
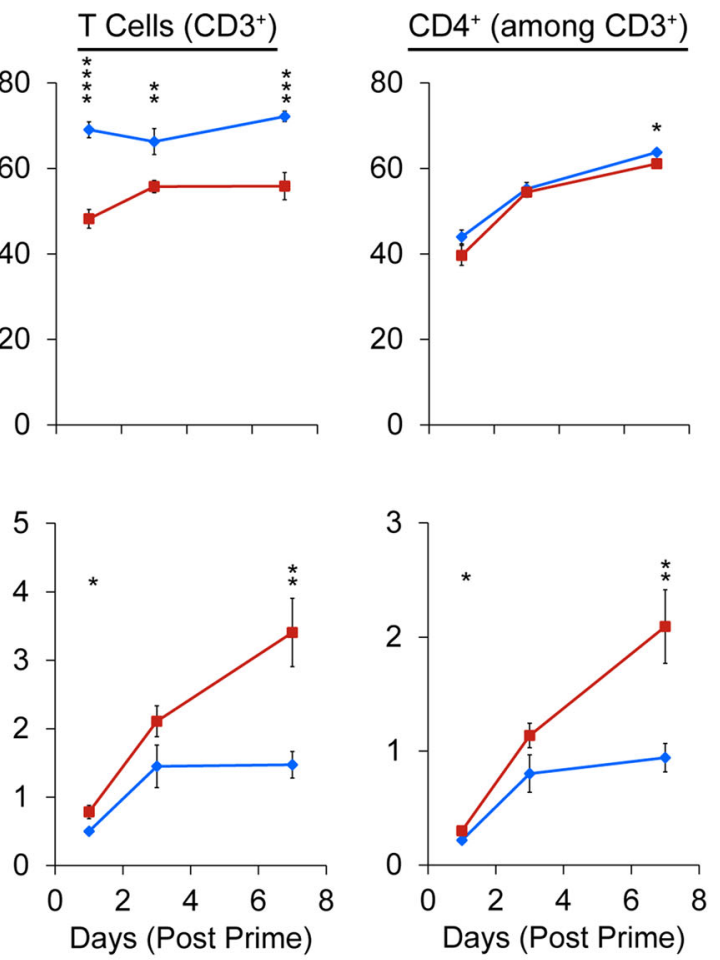

$\rightarrow$ Sham $=$ PolyIC MPIOVA
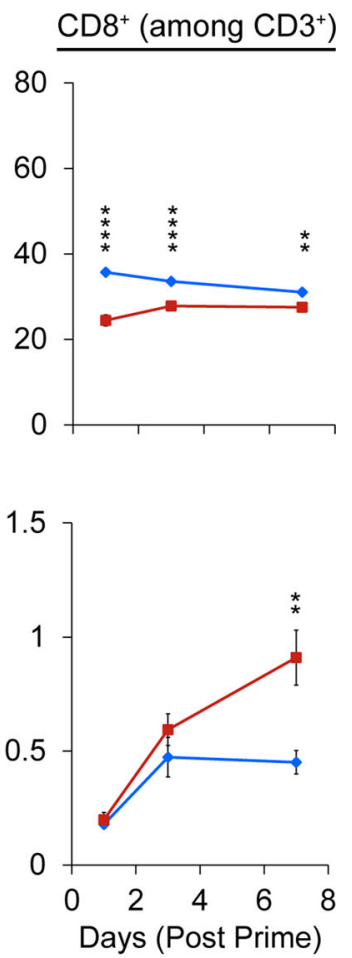

FIGURE 3. i.LN. injection of PolyIC MP/OVA depots increases total number of $\mathrm{T}$ and $\mathrm{B}$ lymphocytes within LNs. (a) Percentages

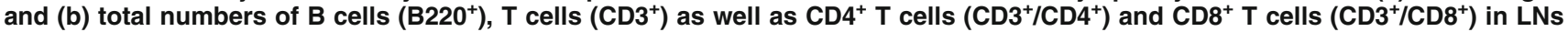
after $i . L N$. injection of PolyIC MP/OVA depots or a sham injection of PBS at days 1, 3, and 7. Numbers are counted in an identical acquisition volume $(80 \mu \mathrm{L}) . n=9-10$ LNs per group with bars depicting mean \pm SEM. $\left({ }^{*} p<0.05 ;{ }^{* *} p<0.01\right.$; ${ }^{\star * *} p<0.001$; $\left.{ }^{\star * \star *} p<0.0001\right)$.

of each LN (discussed below), with nodes treated with PolyIC MP/OVA exhibiting significantly more cells per $\mathrm{LN}$ than the sham at day $1(p<0.01)$; a similar trend was observed over 1 week. In investigating how PolyIC MP/OVA treatment influenced innate immune cell populations, we discovered the frequency of DCs $\left(\mathrm{CD} 11 \mathrm{c}^{+}\right)$did not significantly change over 1 week, while a slight elevation in macrophage $\left(\mathrm{F} 4 / 80^{+}\right)$frequency was observed (Fig. 2c). However, the number of each of these cell types (normalized to equivalent tissue cell suspensions) increased over time, with significantly more DCs $(p<0.001)$ and macrophages $(p<0.01)$ accumulating in the LNs over 7 days following PolyIC MP/OVA injection (Fig. 2d). Similarly, we observed modest changes in the frequency of lymphocytes in the $\mathrm{B}$ cell $\left(\mathrm{B} 220^{+}\right)$and $\mathrm{T}$ cell $\left(\mathrm{CD}^{+}{ }^{+}\right.$; $\left.\mathrm{CD}^{+} / \mathrm{CD}^{+} ; \mathrm{CD}^{+} / \mathrm{CD}^{+}\right)$compartments relative to sham injections (Fig. 3a). However, enumeration of the number of lymphocytes again revealed PolyIC MP/ OVA increased the number of cells in each population, with the maximum difference between groups occurring 7 days after the immunization. Immunohistochemical staining of the LNs at 1 day (Fig. 4a) and 7 days (Fig. 4b) after injection confirmed the increased total number of cells, indicated by the increased area evident in each section; all sections are presented at the same scale. These studies also qualitatively confirmed the increased DC levels we measured in response to PolyIC MP/OVA treatment relative to sham, and the increase in DC number as a function of time. These trends are illustrated in the insets of Fig. $4 \mathrm{~b}$ at day 7 (i.e., sham vs. PolyIC MP/OVA) and the insets of Figs. $4 \mathrm{a}$ and $4 \mathrm{~b}$ for PolyIC MP/OVA (i.e., day 1 vs. day 7), respectively.

\section{PolyIC MP/OVA Treatment Activates LN-Resident $A P C s$}

After determining that i.LN. treatment with PolyIC MP/OVA increases the number of APCs, we tested if these populations exhibited an increased activation state by staining for surface activation markers associated with co-stimulation and antigen presentation (i.e., CD40, CD80, CD86, I-A/I-E). In all cases, PolyIC MP/OVA caused a significant increase in the number of cells positive for each marker compared to the sham injected control (Fig. 5a). Interestingly, the number of activated DCs increased over time with the 


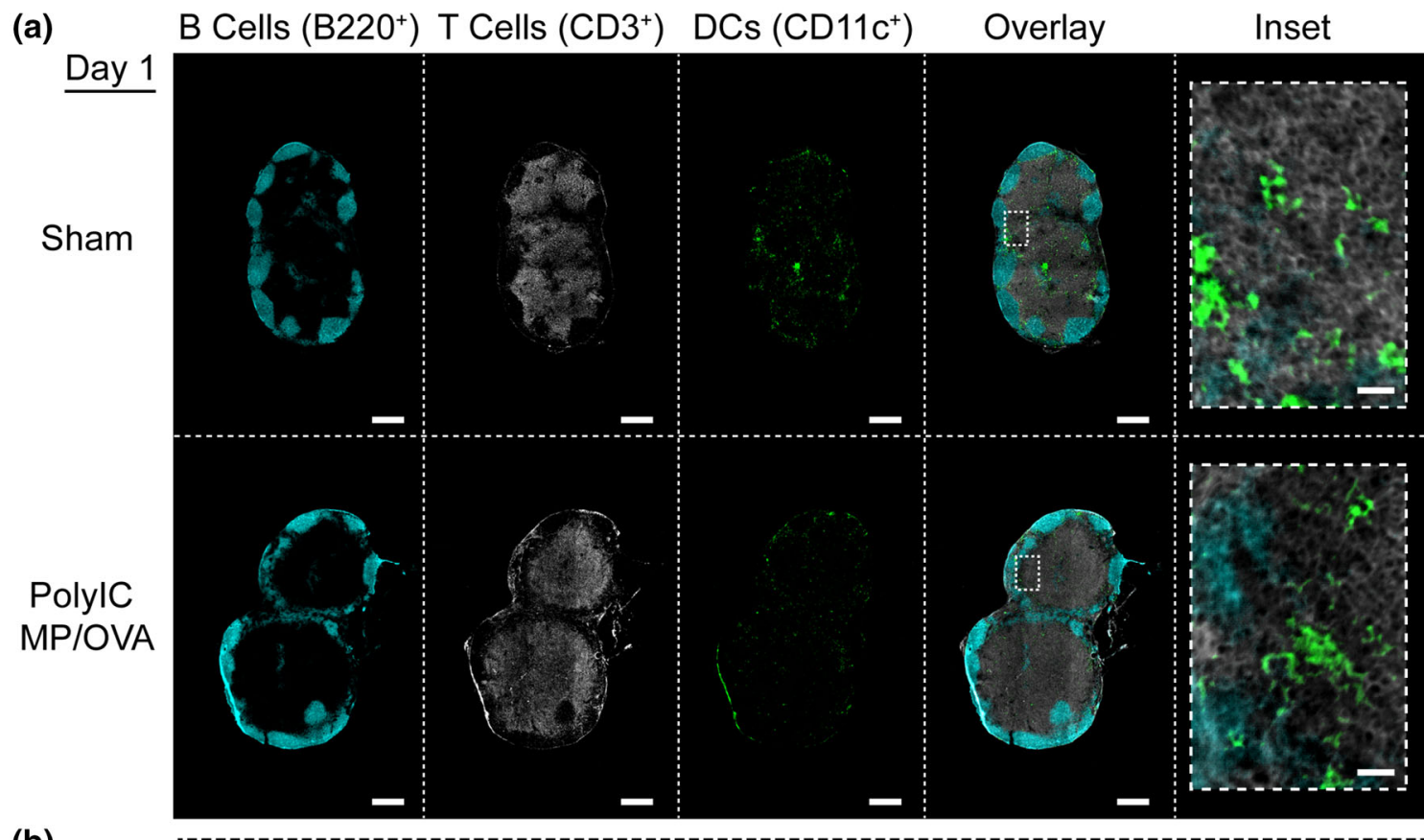

(b)

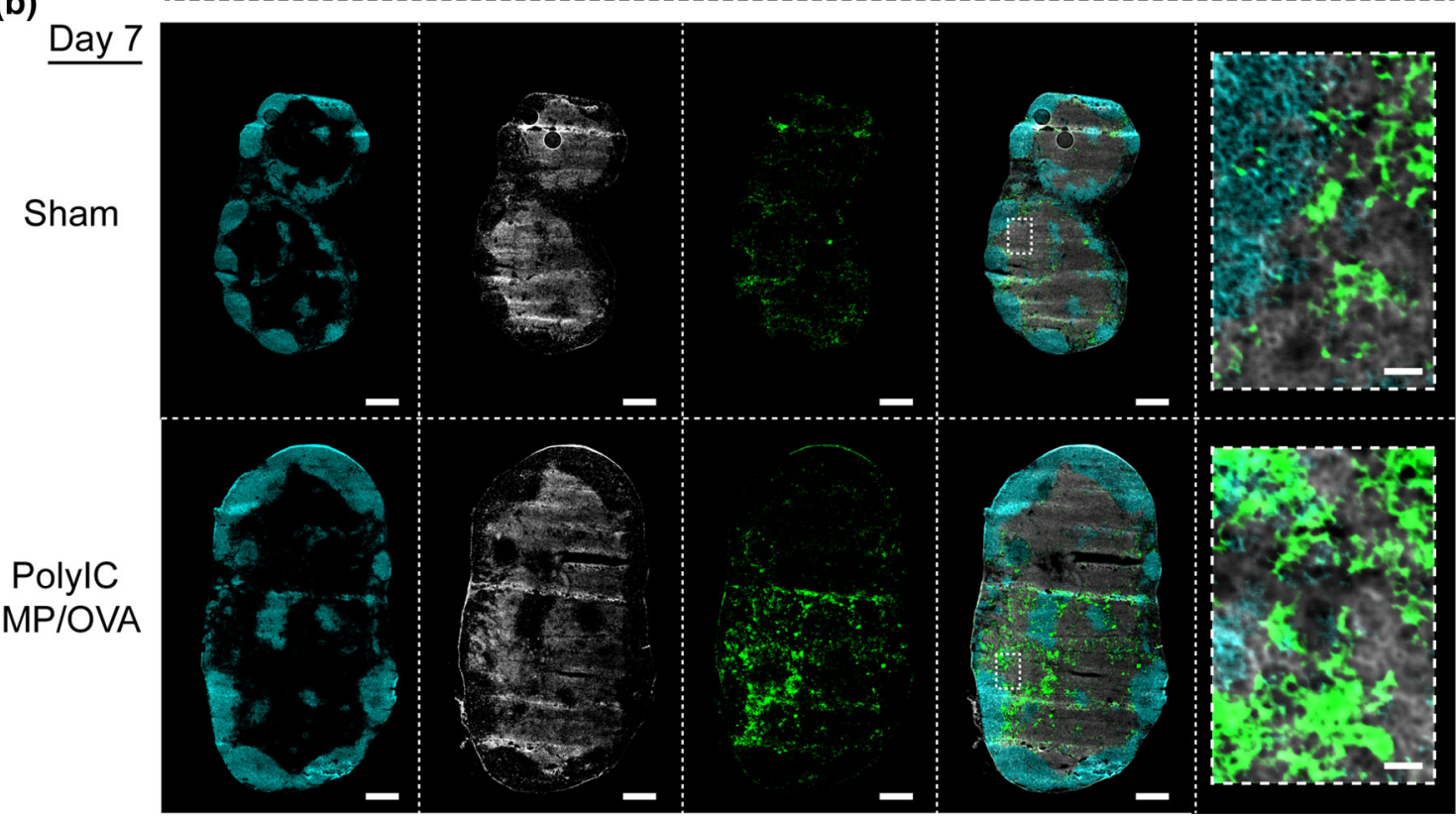

FIGURE 4. Increased LN size and DC numbers in LNs occurs by day 7 after i.LN. injection of PolyIC MP/OVA depots. Histological staining of LNs for B cells (B220 ${ }^{+}$, cyan), T cells (CD3 ${ }^{+}$, white), and DCs (CD11c ${ }^{+}$, green) in LNs 1 day (a) and 7 days (b) after $i . L N$. injection of PolyIC MP/OVA depots or a sham injection of PBS. Scale bar $=400 \mu \mathrm{m} ; 20 \mu \mathrm{m}$ in inset.

highest levels of each marker occurring 7 days after treatment (Fig. 5a, red). The macrophage population exhibited similar activation effects (Fig. 5b). However, compared to DCs, which showed increases in the number of cells expressing each marker over time, only CD40 and I-A/I-E increased as a function of time. Macrophage expression levels of CD80 and CD86-while higher than levels in sham-injected nodes - remained at a near-constant, elevated level over 1 week.

Local Changes in APC Function Drive Local and Systemic Antigen-Specific CD ${ }^{+} T$ Cell Response

We next used MHC-I tetramer staining to investigate if the local activation we observed drove genera- 

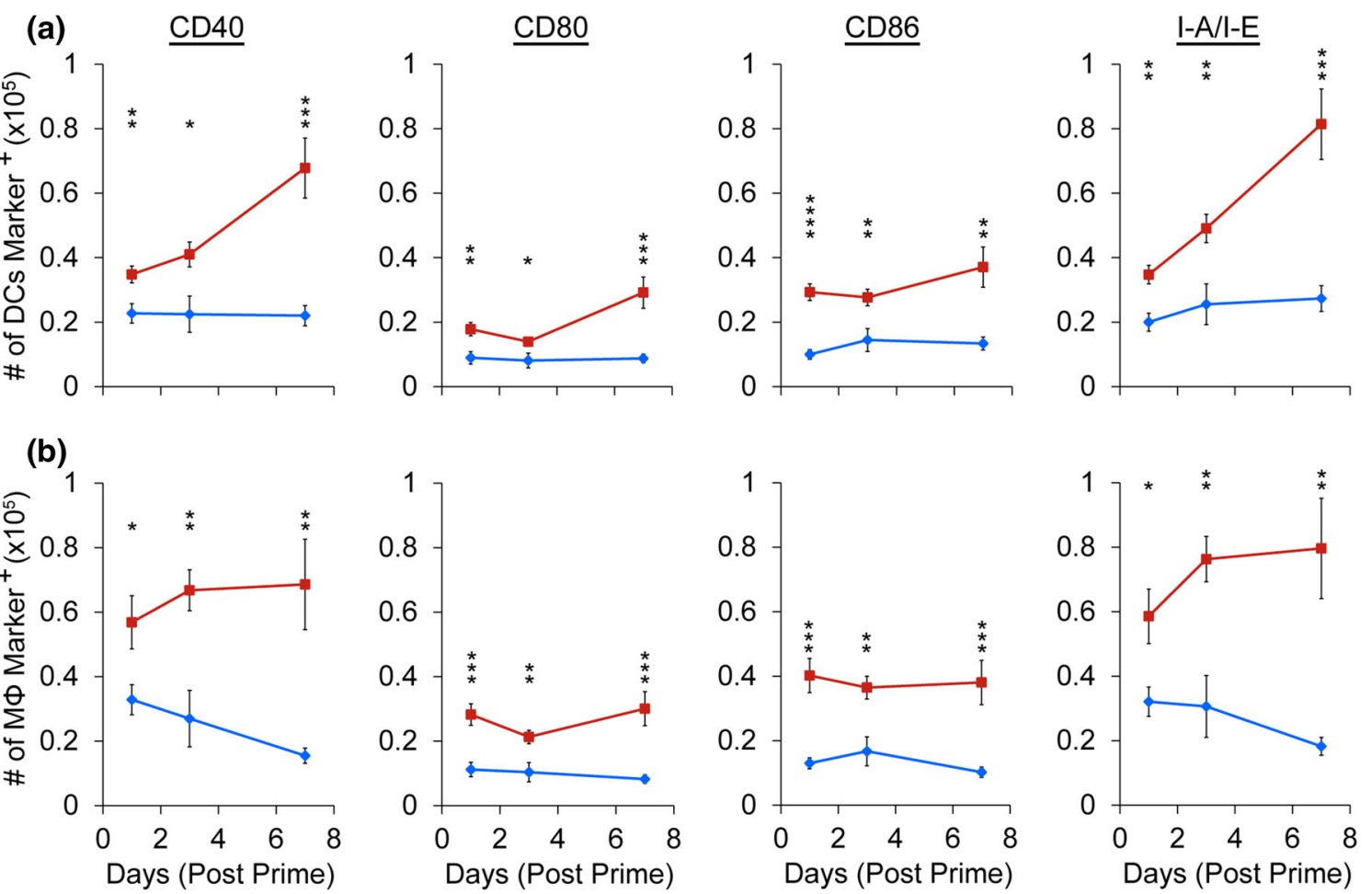

FIGURE 5. PolyIC MP/OVA depots injected i.LN. drive prolonged increase in surface activation marker expression in DCs and macrophages. Number of DCs (a) and macrophages (b) in LNs expressing activation markers CD40, CD80 CD86 and I-A/I-E at 1, 3, and 7 days after $i . L N$. injection of depots. Numbers are counted in an identical acquisition volume (80 $\mu$ L). $n=9-10$ LNs per group with bars depicting mean \pm SEM. $\left({ }^{\star} p<0.05 ;{ }^{* \star} p<0.01 ;{ }^{* \star *} p<0.001 ;{ }^{* \star * *} p<0.0001\right)$.

tion of antigen-specific $\mathrm{T}$ cells, both in treated nodes and systemically. Analysis of LNs after treatment revealed that vaccinating with PolyIC MP/OVA increased both the frequency and number of antigenspecific $\mathrm{CD} 8{ }^{+} \mathrm{T}$ cells within the LN (Figs. 6a and 6b). While the sham injection (Figs. 6a and 6b, blue) remained at a constant, low level, the PolyIC MP/OVA treated mice exhibited a significant $(p<0.01)$ increase in SIINFEKL-specific T cells 7 days after priming. To investigate how these local changes to the $\mathrm{LN}$ microenvironment impacted systemic changes in antigen-specific responses, mice were treated with either PolyIC MP/OVA, empty MPs, a sham injection, or left untreated. After vaccination on Day 0, blood was collected weekly and SIINFEKL tetramer staining was used to determine the percentage of antigen-specific $\mathrm{CD}^{+} \mathrm{T}$ cells circulating in peripheral blood. Figures $6 \mathrm{c}-6 \mathrm{f}$ depicts representative flow cytometry plots showing the gating scheme applied to samples from naïve (Fig. 6c, gray), sham (Fig. 6d, blue), empty MP (Fig. 6e, green), or PolyIC MP/OVA (Fig. 6f, red) treated mice 7 days after immunization. The average SIINFEKL tetramer levels revealed that treatment with PolyIC MP/OVA significantly increased $(p<0.0001)$ systemic levels of SIINFEKL-specific
$\mathrm{CD}^{+} \mathrm{T}$ cells 7 days after treatment, followed by a prototypical contraction period through day 28 (Fig. 6g). The elevated level of SIINFEKL-specific CD ${ }^{+} \mathrm{T}$ cells at day 28 suggested development of immune memory, which we assessed using common markers for effector $\mathrm{T}$ cells and memory $\mathrm{T}$ cells among $\mathrm{CD}^{+} /$Tetramer $^{+}$cells. These studies revealed a nearly twofold increase in the percentage of central memory $\mathrm{T}$ cells $\left(\mathrm{CD} 62 \mathrm{~L}^{\text {high }} / \mathrm{CD} 44^{\text {high }}\right.$ among SIINFEKL-specific $\mathrm{CD} 8^{+}$) and a subsequent decrease in effector memory phenotypes (CD62 $\mathrm{L}^{\text {low }} / \mathrm{CD} 44^{\text {high }}$ ) over this same time (Fig. 6h).

To test the robustness and modularity of this platform, we next tested if $i . L N$. injection expands antigenspecific $\mathrm{T}$ cells with vaccines containing different TLRas or other antigens, in particular, Trp2 peptide - a clinically-relevant tumor associated antigen conserved in murine and human melanoma. ${ }^{38}$ Depots were formulated with either PolyIC or $\mathrm{CpG}-\mathrm{a}$ potent adjuvant being studied to induce anti-tumor immunity 12,49 - and mixed with soluble OVA or Trp2. Mice were immunized i.LN at day 0 with vaccine depots encapsulating identical doses of adjuvant, and then boosted at day 21 with soluble vaccine components s.c. at the tail base. At days 7 and 28 ( 7 days after the prime and 
(a)

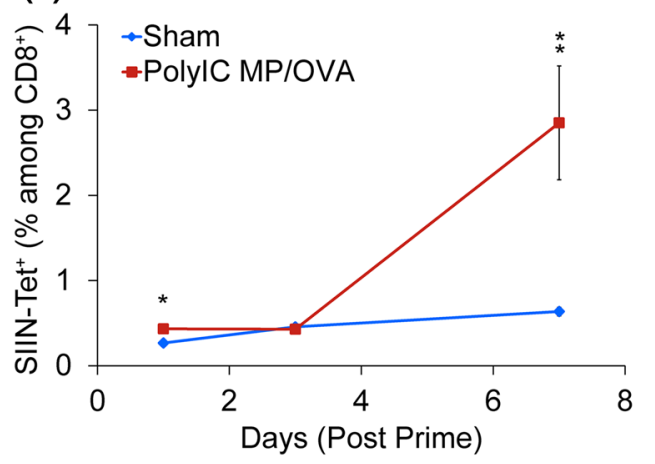

(c)

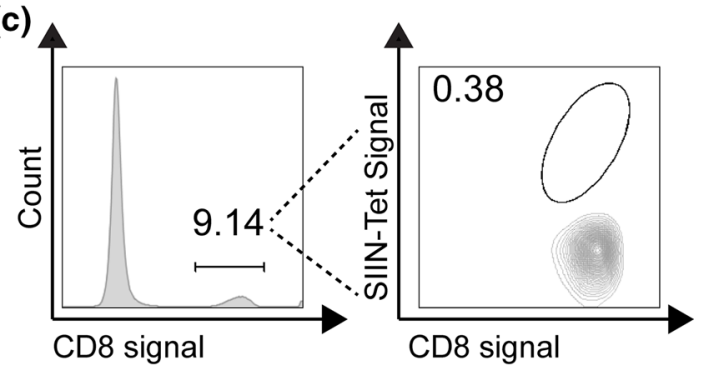

(e)
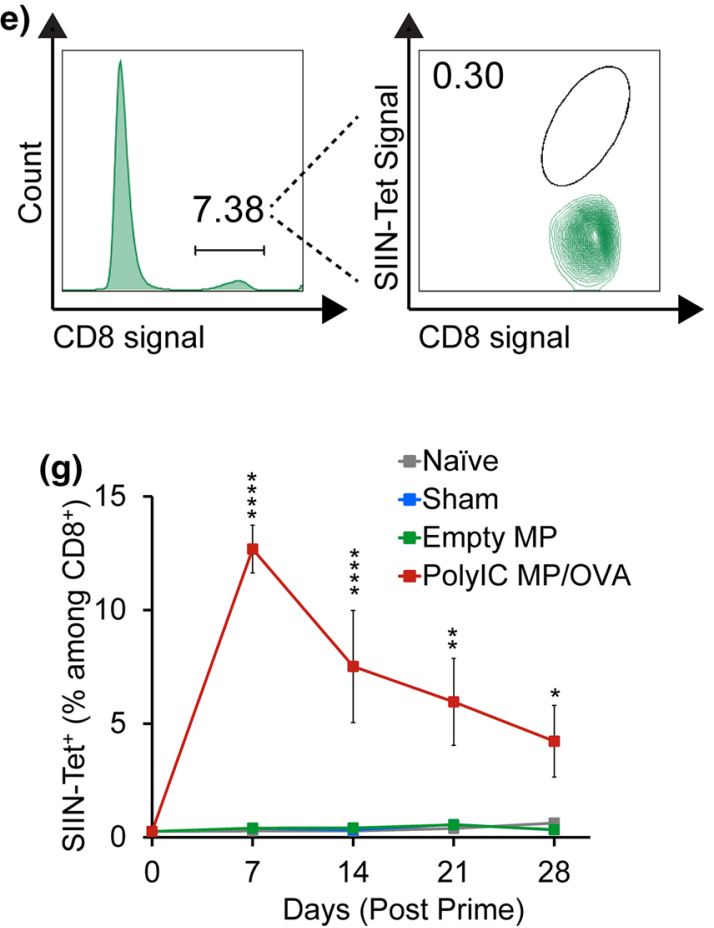

(b)

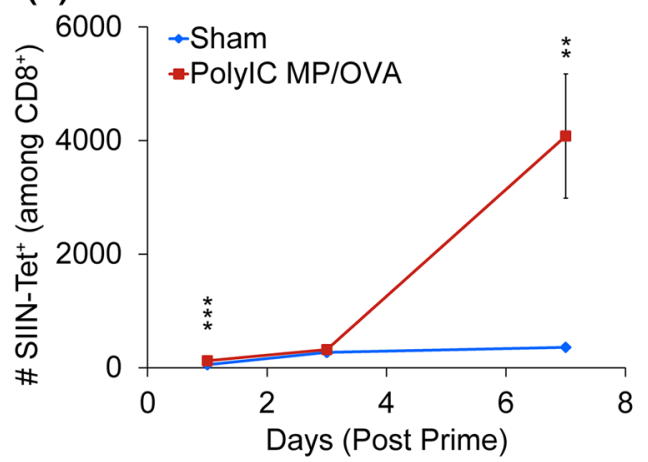

(d)
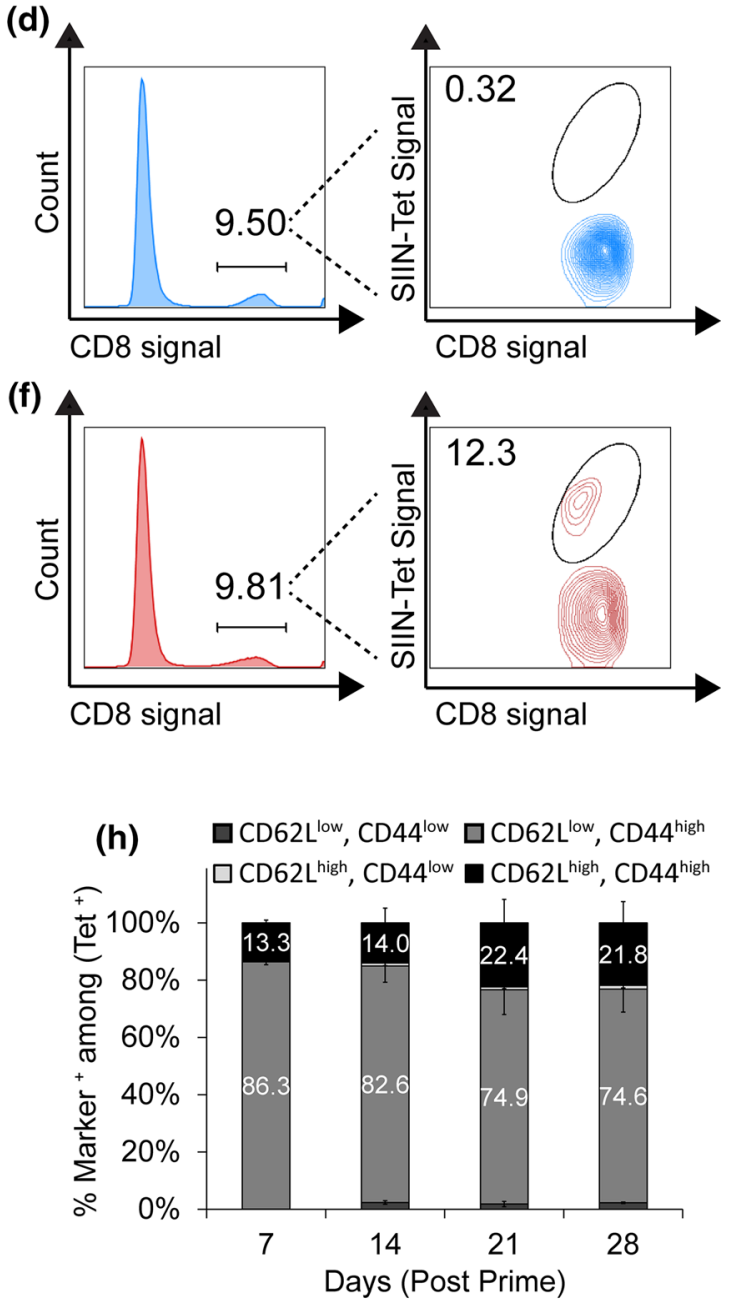

FIGURE 6. i.LN. injection of depots drives antigen-specific T cell responses locally in LNs and systemically in the periphery. (a) Percentage and (b) numbers of SIINFEKL-tetramer ${ }^{+} \mathrm{CD}^{+} \mathrm{T}$ cells in LNs at 1,3 and 7 days after $i . L N$ injection of PolyIC MP/OVA depots or a PBS sham injection. Numbers are counted in an identical acquisition volume $(80 \mu \mathrm{L})$. $n=9-10 \mathrm{LNs}$ per group with bars depicting mean \pm SEM. $\left({ }^{* \star} p<0.01 ;{ }^{* \star \star} p<0.001\right)$ Mice were immunized $i . L N$. with PolylC MP/OVA depots, Empty MPs, a sham injection of PBS or left untreated (naïve), and leukocytes from peripheral blood were stained for SIINFEKL-tetramer ${ }^{+}$CD8 $^{+}$T cells $^{2}$ weekly starting 7 days after immunization. Representative flow cytometry plots illustrating the gating scheme for SIINFEKL tetramer staining of untreated mice (c), mice immunized i.LN. with a sham injection of PBS (d), Empty MPs (e), or PolyIC MP/OVA depots (f) 7 days after treatment. (g) Mean percentage of SIINFEKL-tetramer positive $T$ cells and (h) percentage of SIINFEKL positive T cells with effector (CD62L ${ }^{\text {low }} / C D 44^{\text {high }}$ ) or memory phenotypes (CD62L ${ }^{\text {high }} / C D 44^{\text {high }}$ ) in mice from treatment groups detailed in (c-f). $n=8$ mice for Day $0, n=10$ mice per group at Day 7 , and $n=4-5$ mice per group for Days $14-28$. $\left({ }^{\star} p<\right.$ $\left.0.05 ;{ }^{* \star} p<0.01 ;{ }^{* \star \star} p<0.001 ;{ }^{* \star \star \star} p<0.0001\right)$. 
(a)
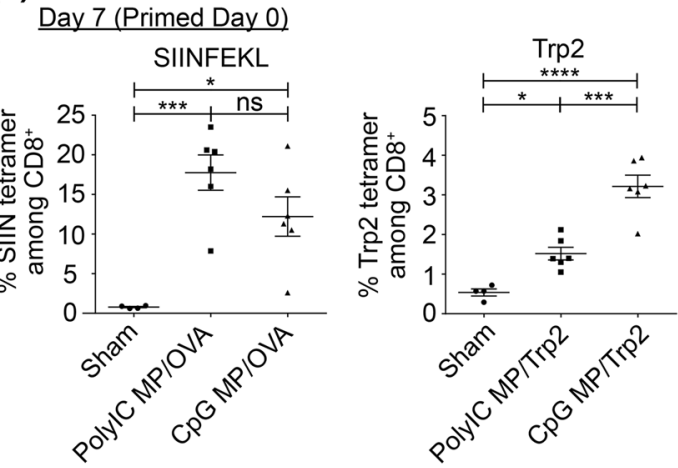

(b)

Day 28 (Boosted Day 21)
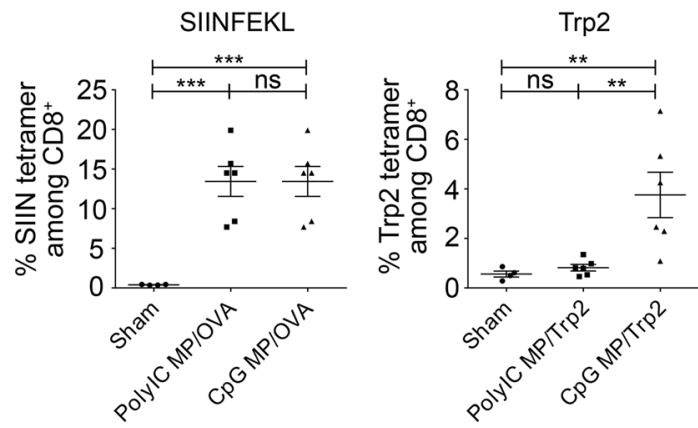

FIGURE 7. CpG MPs induce superior tumor-specific CTL responses compared to PolyIC MPs. Mice were primed at day 0 i.LN. will either PolyIC MPs or CpG MPs, and either a model antigen (OVA) or a melanoma associated antigen (Trp2) in a soluble form. Mice were boosted at day 21, and antigenspecific MHC-I tetramer was used to measure antigen specific $\mathrm{CD8}^{+} \mathrm{T}$ cell responses compared to a sham injection. (a) 7 days after priming, PolyIC and CpG MPs both induced potent levels of SIINFEKL-specific $\mathrm{CD}^{+}$, but no differences were observed as a function of TLRa. In the Trp2 model, both PolyIC and CpG MPs increased the levels of Trp2-specific $\mathrm{CD8}^{+}$T-cells, with $\mathrm{CpG}$ exhibiting a statistically significant increase compared to both the sham and PolylC MP injections. (b) At day 28, 7 days after the boost, a similar response was seen with a robust response in the OVA model for both PolyIC and CpG MPs, but without dependence on the specific TLRa included in the particles. In the Trp2 studies, only CpG MPs induced a significant, potent recall response. $\left({ }^{*} p<0.05\right.$; $\left.{ }^{\star \star} p<0.01 ;{ }^{\star \star \star} p<0.001 ;{ }^{\star \star \star \star} p<0.0001\right)$.

boost injections), peripheral blood was drawn and MHC-I tetramer staining was used to quantify the percentage of antigen specific $\mathrm{CD} 8^{+} \mathrm{T}$ cells $(\mathrm{Trp} 2$ tetramer for Trp2 immunized mice, SIINFEKL tetramer for OVA immunized mice). For mice immunized with OVA vaccine depots both treatments induced very potent antigen-specific responses, but no significant differences were measured between responses induced by $\mathrm{CpG}$ MPs and PolyIC MPs at either day (Fig. 7, left). However, in mice treated with Trp2 vaccine depots, a significantly higher level of Trp2 specific $\mathrm{CD} 8{ }^{+} \mathrm{T}$ cells was observed in mice immunized with $\mathrm{CpG}$ depots compared to PolyIC depots at both time points (Fig. 7, right).

\section{Local administration of $C p G$ particles promotes anti- tumor immunity}

We next used an aggressive melanoma model-B16F10 - to test the functionality of anti-tumor immunity induced by vaccine depots administered by the i.LN route. Since vaccine depots formulated with $\mathrm{CpG}$ promoted superior expansion of Trp2-specific cytotoxic T lymphocytes (CTLs) compared with PolyIC (Fig. 7), we immunized mice with $\mathrm{CpG}$ depots containing $3.5 \mu \mathrm{g}$ of $\mathrm{CpG}$ and suspended in either Trp2, or another conserved melanoma antigen, hgp $100 .^{28,35} \mathrm{In}$ these studies, mice were primed on day 0 with either $\mathrm{CpG} \mathrm{MP} /$ tumor antigen, or as a potent benchmark, $50 \mu \mathrm{g} \mathrm{CpG}$ and tumor antigen emulsified in montanide, one of the strongest adjuvants currently under study. ${ }^{27,54}$ Animals were then boosted on day 15 with identical doses and formulations, but all injections were administered s.c. as a heterologous prime-boost regimen. MHC-I tetramer staining for either Trp2- or hgp100-specific $\mathrm{CD}^{+} \mathrm{T}$ cells revealed formulations containing CpG MPs exhibited significant increases in these populations relative to other groups after both priming and booster injections (Figs. 8a and 8b). After a second boost on day 36 , mice were challenged with B16-F10 metastatic melanoma by implantation of $3 \times 10^{5}$ cells s.c. at the hind flank. Compared to the untreated group (Figs. $8 \mathrm{c}$ and $8 \mathrm{~h}$ ), the mice primed s.c. with montanide/CpG/hgp100 (Figs. $8 \mathrm{~d}$ and $8 \mathrm{~h}$ ) or i.LN. with CpG MPs/hgp100 (Figs. 8f and 8h) did not exhibit any therapeutic gains. In contrast, i.LN immunization with $\mathrm{CpG} \mathrm{MP} / \mathrm{Trp} 2$ slowed tumor growth, resulting in $40 \%$ survival at day 20 (Figs. $8 \mathrm{~g}$ and $8 \mathrm{~h}$ ), while all untreated mice succumbed by this day (Figs. 8c and 8h). Interestingly, while Montanide/ $\mathrm{CpG} /$ Trp2 prolonged survival of mice to 29 days after tumor challenge (Figs. 8e and 8h) the effect appeared less potent than those generated by $\mathrm{CpG}$ MP/Trp2 vaccine regimens, which survived for up to 35 days. The mean survival was $23.0 \pm 4.5$ days for the $\mathrm{CpG}$ $\mathrm{MP} / \mathrm{Trp} 2$ treated group, compared to $20.0 \pm 2.4$ days for the Montanide/CpG/Trp2 treated group, and $16.3 \pm 1.7$ days for the untreated group, further demonstrating the ability of local LN treatment to promote functional, systemic immunity.

\section{DISCUSSION}

Biomaterials offer a robust platform to co-deliver immune signals, target vaccines to specific tissues, and control delivery kinetics. However, most vaccines have 
(a)

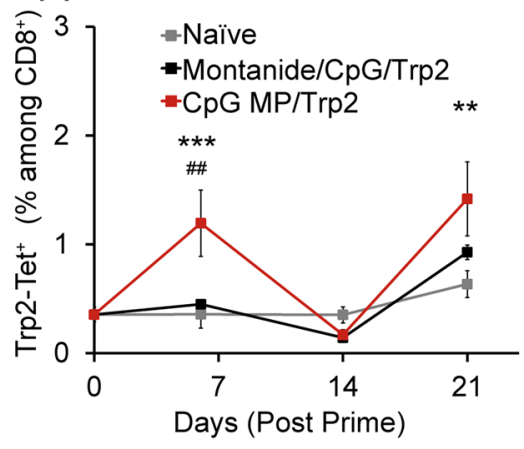

(b)

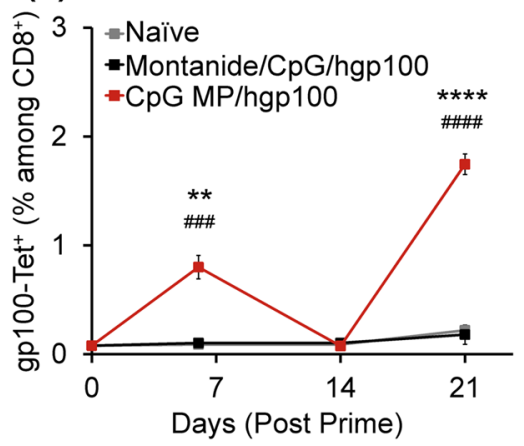

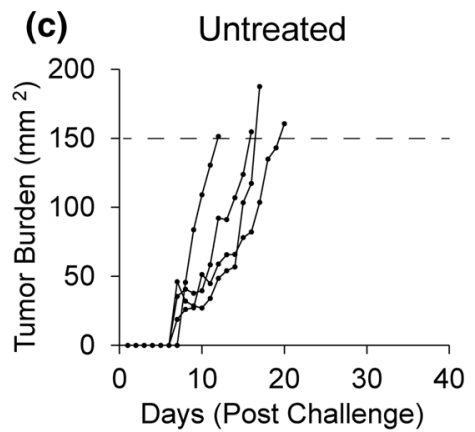

(f) $\mathrm{CpG} \mathrm{MP}+$ soluble hgp100

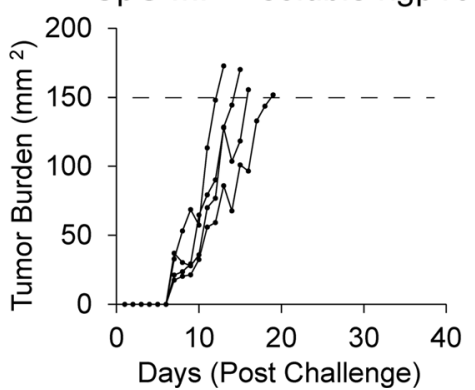

(d) Montanide/CpG/hgp100

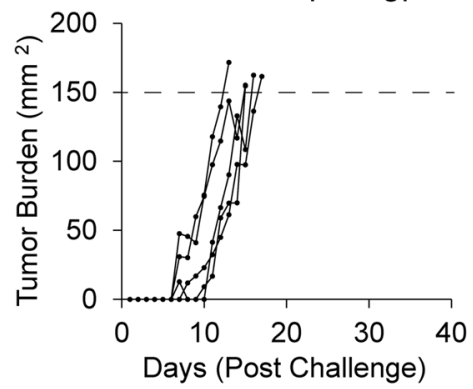

(e) Montanide/CpG/Trp2
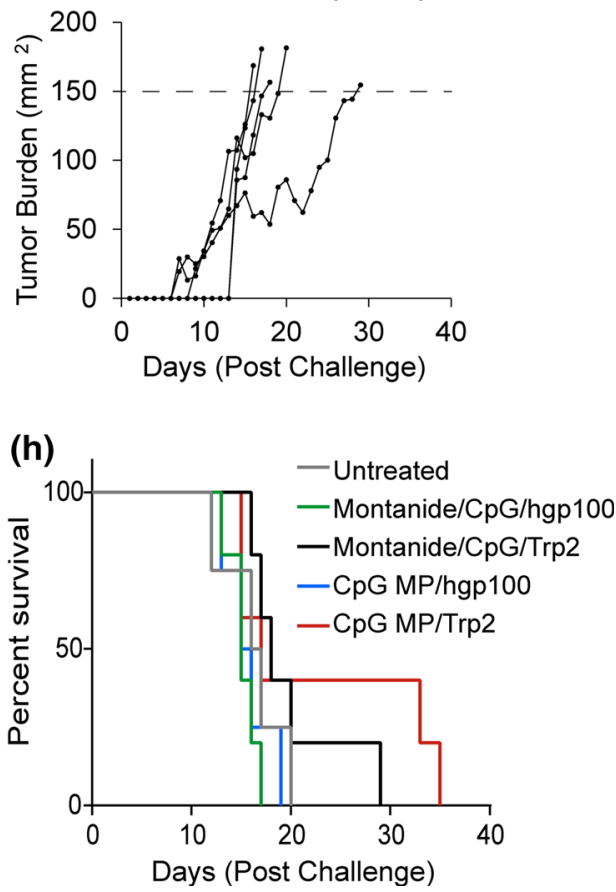

FIGURE 8. i.LN. injection of CpG MP/Trp2 depots promote functional anti-tumor immunity. (a) Mice were left untreated, immunized s.c. with Montanide/CpG/Trp2, or immunized i.LN. with CpG MP/Trp2, followed by s.c. boosts consisting of identical treatments at Day 15. Trp2-tetramer specific $T$ cells were quantified in peripheral blood at 6, 14 and 21 days after immunization. (b) A study conducted using identical treatment regimens as in (a), but including an additional tumor antigen, hgp100. hgp100-specific $\mathrm{CD}^{+} \mathrm{T}$ cell responses in peripheral blood were quantified using hgp100 MHC-I tetramer in peripheral blood at 6, 14 and 21 days after immunization. Values indicate mean \pm SEM. $\left({ }^{\star \star} p<0.01 ;{ }^{\star \star \star} p<0.001 ;{ }^{* \star \star \star} p<0.0001\right.$ between CpG MP groups and naïve;

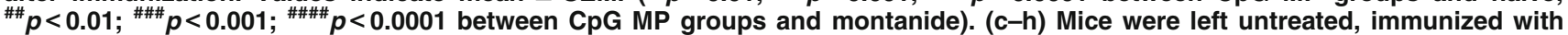
Montanide/CpG/hgp100, Montanide/CpG/Trp2, CpG MP/hgp100 (i.LN.), or CpG MP/Trp2 (i.LN.) followed by s.c. boosts at Day 15 and Day 36 as described in the methods. 43 days after the priming injection, mice were challenged with B16-F10 melanoma. Individual tumor traces of untreated mice (c), mice immunized with Montanide/CpG/hgp100 (d), Montanide/CpG/Trp2 (e), CpG MP/hgp100 (f) and CpG MP/Trp2 (g). (h) Percent survival of mice in the groups shown in (c-g).

complex formulations with multiple components, and understanding how each component influences the immune response alone or together has been challenging thus far. Previous research has shown that altering material properties can influence and improve the targeting of vaccines to LNs through lymphatic drainage or trafficking within specific APCs after internalization. $^{20,30,40,41,49}$ i.LN. delivery, however, of- fers a unique opportunity to directly study how the form and combination of signals that ultimately reach LNs impact immune response without the complexities that occur after vaccines are administered by traditional routes. For example, even efficacious vaccines only result in a small fraction of the injected dose reaching the $\mathrm{LN}$ and spleen - as little as $0.1 \%$, whereas pre-clinical and clinical trials studying $i . L N$. delivery of 
soluble vaccines have demonstrated dose-sparing factors as high as $10^{6}$ relative to common peripheral injection routes. ${ }^{23,45,53}$ With respect to nanoparticles, past studies have revealed that particles administered along common peripheral routes drain to LNs most efficiently when the diameters are in the range of 20$30 \mathrm{~nm}$, whereas even $100 \mathrm{~nm}$ particles drain an order of magnitude less efficiently. ${ }^{42}$ MP drainage relies heavily on APC trafficking. ${ }^{2}$ Our own past findings demonstrate that improved retention of adjuvant in LNs achieved by encapsulation in MPs too large to freely drain from LNs after i.LN. injection drives very strong $\mathrm{T}$ cell responses compared to equivalent doses of soluble adjuvant administered i.LN., or adjuvant MPs administered peripherally (e.g., in muscle). ${ }^{22}$ In contrast, nanoparticles or soluble adjuvant are retained in LNs at intermediate and low levels, respectively, driving correspondingly lower responses relative to MPs. ${ }^{22}$ Thus, here we used i.LN. injection of MPs to add new understanding of how these local treatments alter LN function over time, and how this local evolution impacts systemic immunity.

With respect to local changes in LNs, several of our findings together suggest an adjuvant mechanism underpinned by increased activation of LN-resident APCs. First, we generally observed large difference in the number of immune cells in treated nodes relative to sham injections, with more modest differences in the relative cell compositions. These frequencies - for both innate and adaptive immune cells - were similar to those previously reported in LNs of C57BL6 mice. ${ }^{34}$ Second, we observed persistence of fluorescent MPs for at least 4 weeks (Fig. 1b), and increased activation of LN-resident APCs (e.g., macrophages, DCs) as soon as 1 day after injection. Thus, one important role for the depots appears to be enhanced local APC function that could help increase lymphocyte proliferation and infiltration. The resulting antigen-specific responses showed enhancements consistent with strong $\mathrm{T}$ cell response. For example, OVA-specific T cells developed locally in LN over 7 days, by which time a dramatic increase was measured in peripheral blood. This evolution is consistent with primed lymphocytes migrating out of the LNs as they expand against SIINFEKL presented in these sites. ${ }^{56}$ Similarly, a shift towards a central memory phenotype and away from effector response was also observed over time, a goal for effective vaccines. ${ }^{39}$ Interestingly, we did observe that both depots and sham injections caused modest-sometimes, transient-increases in the frequency of $\mathrm{B}$ cells and $\mathrm{CD} 4{ }^{+} \mathrm{T}$ cells. Thus, an additional enhancing mechanism could be mild inflammation caused by injection that, for example, could upregulate adhesion molecules (e.g., P-, E-selectin) to better retain circulating $\mathrm{T}$ and $\mathrm{B}$ cells. The absence of toxicity, and the intact follicular structure of LNs after either sham or adjuvant MP treatment, further supports the compatibility of this strategy for fundamental or applied uses.

The link between the kinetics of vaccine dosing and induction of immune response is well established, with elegant studies demonstrating that increasing dosing regimens drive synergistic immune responses more effectively than equivalent doses administered in a bolus or at evenly spaced equal doses. ${ }^{24}$ This discovery supports the basic premise for delivery of controlled release depots to LNs, as the local dose of vaccine components locally increases in LNs as cargo is released from degrading polymer particles. ${ }^{22}$ Further, while there is significant potential made possible by determining whether vaccine particles loaded with antigen, adjuvant, or both might be most potent for a particular vaccine, ${ }^{26}$ design of adjuvant-loaded particles offer the appeal of "plug-n-play" vaccination whereby the particle is simply mixed with a soluble adjuvant of interest.

We found i.LN. injection of adjuvant MPs drove antigen-specific $\mathrm{T}$ cell responses against both model antigen (i.e., OVA) and tumor-associated antigens (i.e., Trp2, gp100) mixed with the depots. Interestingly, for OVA, both PolyIC-loaded and CpG-loaded depots performed equivalently, while $\mathrm{CpG}$ was more effective in generating responses against tumor-associated antigens. $\mathrm{CpG}$ has stimulated great interested in preclinical cancer studies owing to effective priming of CTL response. ${ }^{11,12,21,31,49}$ Thus, we benchmarked i.LN. delivery of $\mathrm{CpG}$ MPs mixed with common conserved melanoma antigens, against these same antigens emulsified with $\mathrm{CpG}$ and montanide, one of the strongest vaccine formulations under study. ${ }^{27,54} \mathrm{With}$ respect to both tumor-specific $\mathrm{T}$ cell expansion and anti-tumor immunity, i.LN. depots were superior to montanide, but interestingly, the dose of $\mathrm{CpG}$ in $\mathrm{MP}$ formulations $(3.5 \mu \mathrm{g} / \mathrm{LN})$ was 14 -fold lower than the $50 \mu \mathrm{g}$ dose of $\mathrm{CpG}$ emulsified in the montanide vaccines. Thus, although the efficacy achieved with i.LN. depots in this study was modest $(\sim 40 \%$ of mice exhibited significantly increased survival), the enhanced performance compared with montanide and this dose-sparing supports the potential of future MPbased vaccines administered to LNs.

There are some considerations that might account for the limited efficacy observed in tumor challenge studies. First, the chosen melanoma model is highly aggressive. Second, general features of the tumor microenvironment likely limit immunogenicity, including suppression and antigen editing that prevents tumor-specific CTLs from maintaining function or recognizing antigens in tumors. ${ }^{33,44}$ Third, in our experiments, we observed much higher frequencies of 
SIINFEKL-specific T cell responses after a single i.LN. immunization with OVA depots relative to either melanoma antigen, even after the latter were administered in several booster injections. OVA is a foreign antigen, whereas Trp2 and hgp100 are self-antigens and typically much less immunogenic. Since crosspresentation of minimal epitope peptides such as Trp2 and hgp100 - can enhance immunogenicity, ${ }^{16,18,29,32}$ encapsulation of antigen in MPs alone, or in conjunction with adjuvant might offer one route to further improve potency. However, since significant populations of antigen-specific $\mathrm{CD} 8{ }^{+} \mathrm{T}$ cells were generated against either tumor antigen, we speculate more robust responses might improve effectiveness. Along these lines, recent pre-clinical and clinical studies reveal simultaneously activating multiple TLR pathways during cancer therapy can enhance therapeutic efficacy, ${ }^{1,5,13,52}$ suggesting another strategy based on loading of MPs with multiple TLRas.

i.LN. delivery of MPs also provides some unique opportunities to impact the tumor microenvironment through appropriate selection of the $\mathrm{LN}$ for injection. In our studies we selected the inguinal LN for ease of injection based on our past work, and what has been used in recent human trials involving i.LN. delivery of soluble tumor antigens to inguinal LNs. ${ }^{43}$ However, this technique could also be used to target tumor draining lymph nodes (TDLN), sites which have recently been shown to be effective for passive targeting of cancer vaccines. ${ }^{9,10,20,49}$ Remarkably, several landmark studies also demonstrate that both anti-tumor $\mathrm{T}$ cells and regulatory $T$ cells $\left(\mathrm{T}_{\text {REGs }}\right)$ - cells that suppress antitumor response in tumors - are primed in the same LN ${ }^{8,17}$ Thus, direct LN targeting of TDLNs might allow local polarization toward effector cells while also reducing suppressive $T_{\text {REGs }}$ that play an important role in maintaining the suppressive tumor microenvironment. This may further provide an opportunity to effectively combat tumors without affecting natural regulatory activity in other distant LNs. It is also possible that targeting TDLNs is not necessary if optimized particles expand tumor-specific cells that are able to migrate to tumors, but further studies will be needed to investigate this possibility. Finally, creating opportunities to overcome the suppressive characteristics of tumors by directly targeting the TDLN, or pairing with exciting new immunotherapies such as checkpoint blockades could also have offer significant potential for cancer vaccination. ${ }^{36,46}$

\section{CONCLUSION}

i.LN. injection allows direct control over the dose and combinations of materials administered to LNs, supporting a new approach for studying the impact of vaccines on the LN microenvironment. Here, we demonstrate that a single i.LN. injection can lead to dramatic local changes in these tissues, increasing the number and function of both APCs and lymphocytes. The local changes result in systemic, but antigenspecific pro-immune function that provides functional anti-tumor immunity in a melanoma model. Thus, this approach might hold clinical utility for vaccines based on intra-LN controlled release of antigens and adjuvants, while also providing a strategy to evaluate the immunogenicity of biomaterial carriers themselves, or to design carriers loaded with defined combinations of antigens and adjuvants.

\section{ACKNOWLEDGMENTS}

This work was supported in part by NSF CAREER Award \# 1351688, the Damon Runyon Foundation (\# DRR3415), the Melanoma Research Alliance (\# 348963), Alex's Lemonade Stand (\# 27120), the Alliance for Cancer Gene Therapy (\# 15051543), and National Multiple Sclerosis Society Award \# RG1501-02968 and \# PP2103. J.I.A. is a trainee on NIH Grant \# T32 AI089621 and a Graduate Fellow supported by the American Association of Pharmaceutical Scientists Foundation. J.M.G. is a grantee of the Pediatric Oncology Student Training Award (\# 15082537) from Alex's Lemonade Stand Foundation. L.H.T. is an NSF Graduate Fellow (\# DGE1322106). C.M.J. is a Damon-Runyon Rachleff Innovator supported by the Damon Runyon Foundation.

\section{CONFLICT OF INTEREST}

James I. Andorko, Joshua M. Gammon, Lisa H. Tostanoski, Qin Zeng, and Christopher M. Jewell declare that they have no conflicts of interest.

\section{ETHICAL STANDARDS}

No human studies were carried out by the authors of this article. All institutional and national guidelines for the care and use of laboratory animals were followed and approved by the appropriate institutional committees.

\section{OPEN ACCESS}

This article is distributed under the terms of the Creative Commons Attribution 4.0 International License (http://creativecommons.org/licenses/by/4.0/), 
which permits unrestricted use, distribution, and reproduction in any medium, provided you give appropriate credit to the original author(s) and the source, provide a link to the Creative Commons license, and indicate if changes were made.

\section{REFERENCES}

${ }^{1}$ Alpizar, Y. A., B. Chain, M. K. Collins, J. Greenwood, D. Katz, H. J. Stauss, et al. Ten years of progress in vaccination against cancer: the need to counteract cancer evasion by dual targeting in future therapies. Cancer Immunol Immunother 60:1127-1135, 2011.

${ }^{2}$ Andorko, J. I., K. L. Hess, and C. M. Jewell, Harnessing biomaterials to engineer the lymph node microenvironment for immunity or tolerance. AAPS J. 17:323-338, 2015.

${ }^{3}$ Andorko, J. I., K. L. Hess, K. G. Pineault, and C. M. Jewell. Intrinsic immunogenicity of rapidly-degradable polymers evolves during degradation. Acta Biomater. 32:24-34, 2016.

${ }^{4}$ Andorko, J. I., L. H. Tostanoski, E. Solano, M. Mukhamedova, and C. M. Jewell. Intra-lymph node injection of biodegradable polymer particles. J. Vis. Exp. 83:e50984, 2014.

${ }^{5}$ Aranda, F., E. Vacchelli, F. Obrist, A. Eggermont, J. Galon, C. Sautes-Fridman, et al. Trial watch: toll-like receptor agonists in oncological indications. Oncoimmunology 3:e29179, 2014.

${ }^{6}$ Bagchi, A., E. A. Herrup, H. S. Warren, J. Trigilio, H. S. Shin, C. Valentine, et al. Myd88-dependent and myd88independent pathways in synergy, priming, and tolerance between tlr agonists. J. Immunol. 178:1164-1171, 2007.

${ }^{7}$ Barria, M. I., J. L. Garrido, C. Stein, E. Scher, Y. C. Ge, S. M. Engel, et al. Localized mucosal response to intranasal live attenuated influenza vaccine in adults. J. Infect. Dis. 207:115-124, 2013.

${ }^{8}$ Colombo, M. P., and S. Piconese. Regulatory t-cell inhibition versus depletion: the right choice in cancer immunotherapy. Nat. Rev. Cancer 7:880-887, 2007.

${ }^{9}$ Crompton, J. G., D. Clever, R. Vizcardo, M. Rao, and N. P. Restifo. Reprogramming antitumor immunity. Trends Immunol. 35:178-185, 2014.

${ }^{10}$ Crompton, J. G., M. Sukumar, and N. P. Restifo. Uncoupling t-cell expansion from effector differentiation in cell-based immunotherapy. Immunol. Rev. 257:264-276, 2014.

${ }^{11}$ Dankbar, B., K. Neugebauer, C. Wunrau, C. O. Tibesku, A. Skwara, T. Pap, et al. Hepatocyte growth factor induction of macrophage chemoattractant protein-1 and osteophyte-inducing factors in osteoarthritis. J. Orthop. Res. 25:569-577, 2007.

${ }^{12}$ de Titta, A., M. Ballester, Z. Julier, C. Nembrini, L. Jeanbart, A. J. van der Vlies, et al. Nanoparticle conjugation of cpg enhances adjuvancy for cellular immunity and memory recall at low dose. Proc. Natl. Acad. Sci. USA 110:19902-19907, 2013.

${ }^{13}$ Delamarre, L., I. Mellman, and M. Yadav. Cancer immunotherapy. Neo approaches to cancer vaccines. Science 348:760-761, 2015.

${ }^{14}$ Girard, J. P., C. Moussion, and R. Forster. Hevs, lymphatics and homeostatic immune cell trafficking in lymph nodes. Nat. Rev. Immunol. 12:762-773, 2012.
${ }^{15}$ Hanson, M. C., M. P. Crespo, W. Abraham, K. D. Moynihan, G. L. Szeto, S. H. Chen, et al. Nanoparticulate sting agonists are potent lymph node-targeted vaccine adjuvants. J. Clin. Invest. 125:2532-2546, 2015.

${ }^{16}$ Hirosue, S., I. C. Kourtis, A. J. van der Vlies, J. A. Hubbell, and M. A. Swartz. Antigen delivery to dendritic cells by poly(propylene sulfide) nanoparticles with disulfide conjugated peptides: cross-presentation and t cell activation. Vaccine 28:7897-7906, 2010.

${ }^{17}$ Hiura, T., H. Kagamu, S. Miura, A. Ishida, H. Tanaka, J. Tanaka, et al. Both regulatory t cells and antitumor effector $\mathrm{t}$ cells are primed in the same draining lymph nodes during tumor progression. J. Immunol. 175:5058-5066, 2005.

${ }^{18}$ Irvine, D. J., M. A. Swartz, and G. L. Szeto. Engineering synthetic vaccines using cues from natural immunity. Nat. Mater. 12:978-990, 2013.

${ }^{19}$ Irvine, D. J., M. A. Swartz, and G. L. Szeto. Engineering synthetic vaccines using cues from natural immunity. Nat. Mater. 12:978-990, 2013.

${ }^{20}$ Jeanbart, L., M. Ballester, A. De Titta, P. Corthésy, P. Romero, J. A. Hubbell, et al. Enhancing efficacy of anticancer vaccines by targeted delivery to tumor-draining lymph nodes. Cancer Immunol. Res. 2(5):436-447, 2014.

${ }^{21}$ Jerome, V., A. Graser, R. Muller, R. E. Kontermann, and A. Konur. Cytotoxic t lymphocytes responding to low dose trp2 antigen are induced against b16 melanoma by liposome-encapsulated trp2 peptide and cpg DNA adjuvant. $J$. Immunother. 29:294-305, 2006.

${ }^{22}$ Jewell, C. M., S. C. B. Lopez, and D. J. Irvine. In situ engineering of the lymph node microenvironment via intranodal injection of adjuvant-releasing polymer particles. Proc. Natl. Acad. Sci. USA 108:15745-15750, 2011.

${ }^{23}$ Johansen, P., A. C. Haffner, F. Koch, K. Zepter, I. Erdmann, K. Maloy, et al. Direct intralymphatic injection of peptide vaccines enhances immunogenicity. Eur. J. Immunol. 35:568-574, 2005.

${ }^{24}$ Johansen, P., T. Storni, L. Rettig, Z. Qiu, A. Der-Sarkissian, K. A. Smith, et al. Antigen kinetics determines immune reactivity. Proc. Natl. Acad. Sci. USA 105:51895194, 2008.

${ }^{25}$ Joshi, V. B., S. M. Geary, B. R. Carrillo-Conde, B. Narasimhan, and A. K. Salem. Characterizing the antitumor response in mice treated with antigen-loaded polyanhydride microparticles. Acta Biomater. 9:5583-5589, 2013.

${ }^{26}$ Kasturi, S. P., I. Skountzou, R. A. Albrecht, D. Koutsonanos, T. Hua, H. I. Nakaya, et al. Programming the magnitude and persistence of antibody responses with innate immunity. Nature 470:543-U136, 2011.

${ }^{27}$ Kenter, G. G., M. J. Welters, A. R. Valentijn, M. J. Lowik, D. M. Berends-van der Meer, A. P. Vloon, et al. Vaccination against hpv-16 oncoproteins for vulvar intraepithelial neoplasia. N. Engl. J. Med. 361:1838-1847, 2009.

${ }^{28}$ Klebanoff, C. A., L. Gattinoni, D. C. Palmer, P. Muranski, Y. Ji, C. S. Hinrichs, et al. Determinants of successful $\operatorname{cd} 8(+)$ t-cell adoptive immunotherapy for large established tumors in mice. Clin. Cancer Res. 17:5343-5352, 2011.

${ }^{29}$ Kovacsovicsbankowski, M., and K. L. Rock. A phagosome-to-cytosol pathway for exogenous antigens presented on mhc class-i molecules. Science 267:243-246, 1995.

${ }^{30}$ Liu, H. P., K. D. Moynihan, Y. R. Zheng, G. L. Szeto, A. V. Li, B. Huang, et al. Structure-based programming of lymph-node targeting in molecular vaccines. Nature 507:519-522, 2014.

${ }^{31}$ Miconnet, I., S. Koenig, D. Speiser, A. Krieg, P. Guillaume, J. C. Cerottini, et al. Cpg are efficient adjuvants for 
specific ctl induction against tumor antigen-derived peptide. J. Immunol. 168:1212-1218, 2002.

${ }^{32}$ Moon, J. J., H. Suh, A. Bershteyn, M. T. Stephan, H. P. Liu, B. Huang, et al. Interbilayer-crosslinked multilamellar vesicles as synthetic vaccines for potent humoral and cellular immune responses. Nat. Mater. 10:243-251, 2011.

${ }^{33}$ Motz, G. T., and G. Coukos. Deciphering and reversing tumor immune suppression. Immunity 39:61-73, 2013.

${ }^{34}$ Noubade, R., K. Wong, N. Ota, S. Rutz, C. Eidenschenk, P. A. Valdez, et al. Nrros negatively regulates reactive oxygen species during host defence and autoimmunity. Nature 509:235-239, 2014.

${ }^{35}$ Overwijk, W. W., M. R. Theoret, S. E. Finkelstein, D. R. Surman, L. A. de Jong, F. A. Vyth-Dreese, et al. Tumor regression and autoimmunity after reversal of a functionally tolerant state of self-reactive $\mathrm{cd} 8^{+}$T cells. J. Exp. Med. 198:569-580, 2003.

${ }^{36}$ Pardoll, D. M. The blockade of immune checkpoints in cancer immunotherapy. Nat. Rev. Cancer 12:252-264, 2012.

${ }^{37}$ Park, J., and J. E. Babensee. Differential functional effects of biomaterials on dendritic cell maturation. Acta Biomater. 8:3606-3617, 2012.

${ }^{38}$ Parkhurst, M. R., E. B. Fitzgerald, S. Southwood, A. Sette, S. A. Rosenberg, and Y. Kawakami. Identification of a shared hla-a*0201-restricted t-cell epitope from the melanoma antigen tyrosinase-related protein $2(\operatorname{trp} 2)$. Cancer Res. 58:4895-4901, 1998.

${ }^{39}$ Pulendran, B., and R. Ahmed. Immunological mechanisms of vaccination. Nat. Immunol. 12:509-517, 2011.

${ }^{40}$ Randolph, G. J., V. Angeli, and M. A. Swartz. Dendriticcell trafficking to lymph nodes through lymphatic vessels. Nat. Rev. Immunol. 5:617-628, 2005.

${ }^{41}$ Reddy, S. T., A. Rehor, H. G. Schmoekel, J. A. Hubbell, and M. A. Swartz. In vivo targeting of dendritic cells in lymph nodes with poly(propylene sulfide) nanoparticles. $J$. Controll. Release 112:26-34, 2006.

${ }^{42}$ Reddy, S. T., A. J. van der Vlies, E. Simeoni, V. Angeli, G. J. Randolph, C. P. O’Neill, et al. Exploiting lymphatic transport and complement activation in nanoparticle vaccines. Nat. Biotechnol. 25:1159-1164, 2007.

${ }^{43}$ Ribas, A., J. S. Weber, B. Chmielowski, B. Comin-Anduix, D. Lu, M. Douek, et al. Intra-lymph node prime-boost vaccination against melan a and tyrosinase for the treatment of metastatic melanoma: results of a phase 1 clinical trial. Clin. Cancer Res. 17:2987-2996, 2011.

${ }^{44}$ Schreiber, R. D., L. J. Old, and M. J. Smyth. Cancer immunoediting: integrating immunity's roles in cancer suppression and promotion. Science 331:1565-1570, 2011.
${ }^{45}$ Senti, G., P. Johansen, and T. M. Kundig. Intralymphatic immunotherapy. Curr. Opin. Allergy Clin. Immunol. 9:537543, 2009.

${ }^{46}$ Sharma, P., and J. P. Allison. Immune checkpoint targeting in cancer therapy: toward combination strategies with curative potential. Cell 161:205-214, 2015.

${ }^{47}$ Sharp, F. A., D. Ruane, B. Claass, E. Creagh, J. Harris, P. Malyala, et al. Uptake of particulate vaccine adjuvants by dendritic cells activates the nalp3 inflammasome. Proc. Natl. Acad. Sci. USA 106:870-875, 2009.

${ }^{48}$ Slutter, B., S. M. Bal, Z. Ding, W. Jiskoot, and J. A. Bouwstra. Adjuvant effect of cationic liposomes and cpg depends on administration route. J. Controll. Release 154:123-130, 2011.

${ }^{49}$ Thomas, S. N., E. Vokali, A. W. Lund, J. A. Hubbell, and M. A. Swartz. Targeting the tumor-draining lymph node with adjuvanted nanoparticles reshapes the anti-tumor immune response. Biomaterials 35:814-824, 2014.

${ }^{50}$ Timmermans, K., T. S. Plantinga, M. Kox, M. Vaneker, G. J. Scheffer, G. J. Adema, et al. Blueprints of signaling interactions between pattern recognition receptors: implications for the design of vaccine adjuvants. Clin. Vaccine Immunol. 20:427-432, 2013.

${ }^{51}$ Tom, J. K., R. J. Mancini, and A. P. Esser-Kahn. Covalent modification of cell surfaces with tlr agonists improves \& directs immune stimulation. Chem. Commun. 49:96189620, 2013.

${ }^{52}$ Vacchelli, E., A. Eggermont, C. Sautes-Fridman, J. Galon, L. Zitvogel, G. Kroemer, et al. Trial watch: toll-like receptor agonists for cancer therapy. Oncoimmunology 2:e25238, 2013.

${ }^{53}$ Witten, M., H. J. Malling, L. Blom, B. C. Poulsen, and L. K. Poulsen. Is intralymphatic immunotherapy ready for clinical use in patients with grass pollen allergy? J. Allergy Clin. Immunol. 132:1248-1252, 2013.

${ }^{54}$ Wu, T. Y. H., M. Singh, A. T. Miller, E. De Gregorio, F. Doro, U. D'Oro, et al. Rational design of small molecules as vaccine adjuvants. Sci. Transl. Med. 6:263ra160, 2014.

${ }^{55}$ Zhu, Q., C. Egelston, S. Gagnon, Y. J. Sui, I. M. Belyakov, D. M. Klinman, et al. Using 3 tlr ligands as a combination adjuvant induces qualitative changes in $\mathrm{t}$ cell responses needed for antiviral protection in mice. J. Clin. Investig. 120:607-616, 2010

${ }^{56}$ Zinkernagel, R. M., S. Ehl, P. Aichele, S. Oehen, T. Kündig, and H. Hengartner. Antigen localisation regulates immune responses in a dose- and time-dependent fashion: a geographical view of immune reactivity. Immunol. Rev. 156:199-209, 1997. 